\title{
Contrast-enhanced magnetomotive ultrasound imaging (CE-MMUS) for colorectal cancer staging: Assessment of sensitivity and resolution to detect alterations in tissue stiffness
}

\author{
$1^{\text {st }}$ Sandra Sjöstrand \\ Dept. of Biomedical \\ Engineering \\ Lund University \\ Lund, Sweden \\ sandra.sjostrand@ \\ bme.lth.se
}

\author{
$2^{\text {nd }}$ Maria Evertsson \\ Dept. of Clinical Sciences \\ Lund \& Biomedical \\ Engineering \\ Lund University \\ Lund, Sweden \\ maria.evertsson@ \\ med.lu.se
}

\author{
$3^{\text {rd }}$ Claire Thring \\ Biomedical Engineering \\ University of Strathclyde \\ Glasgow, United \\ Kingdom \\ claire.thring@strath.ac.uk
}

\author{
$4^{\text {th }}$ Marion Bacou \\ Edinburgh Cancer \\ Research Centre \\ University of Edinburgh \\ Edinburgh, United \\ Kingdom \\ m.bacou@sms.ed.ac.uk
}

\author{
$5^{\text {th }}$ Susan Farrington \\ Edinburgh Cancer \\ Research Centre \\ University of Edinburgh \\ Edinburgh, United \\ Kingdom \\ susan.farrington@ \\ ed.ac.uk
}

\author{
$6^{\text {th }}$ Susan Moug \\ NHS Greater Glasgow and Clyde \\ Glasgow, United Kingdom \\ susan.moug@nhs.net
}

\author{
$7^{\text {th }}$ Carmel Moran \\ Centre for Cardiovascular \\ Science \\ University of Edinburgh \\ Edinburgh, United Kingdom \\ carmel.moran@ed.ac.uk
}

\author{
$8^{\text {th }}$ Tomas Jansson \\ Dept. of Clinical Sciences Lund \\ Biomedical Engineering \\ Lund University, and Clinical \\ Engineering Skåne, Medical \\ Services, Region Skåne \\ Lund, Sweden \\ tomas.jansson@med.lu.se
}

\author{
$9^{\text {th }}$ Helen Mulvana \\ Biomedical Engineering \\ University of Strathclyde \\ Glasgow, United Kingdom \\ helen.mulvana@strath.ac.uk
}

\begin{abstract}
A key challenge in the treatment of colorectal cancer is identification of the sentinel draining lymph node. Magnetomotive ultrasound, MMUS, has identified lymph nodes in rat models: superparamagnetic iron oxide nanoparticles (SPIONs) accumulated in the lymph are forced to oscillate by an external magnetic field; the resulting axial displacement is recovered allowing structure delineation with potential to indicate alterations in tissue stiffness, but it is limited by small vibration amplitudes. We propose CE-MMUS using SPION loaded microbubbles (SPION-MBs) to enhance sensitivity, reduce toxicity, and offer additional diagnostic or perfusion information. Laser doppler vibrometry measurements was performed on SPION containing tissue mimicking material during magnetic excitation. These measurements show a vibration amplitude of 279 $\pm 113 \mu \mathrm{m}$ in a material with Young's modulus of $24.3 \pm 2.8 \mathrm{kPa}$, while the displacements were substantially larger, $426 \pm 9 \mu \mathrm{m}$, in the softer material, with a Young's modulus of $9.6 \pm 0.8 \mathrm{kPa}$. Magnetic field measurement data was used to calibrate finite element modelling of both MMUS and CE-MMUS. SPION-MBs were shown to be capable of inducing larger tissue displacements under a given magnetic field than SPIONs alone, leading to axial displacements of up to $2.3 x$ larger. A doubling in tissue stiffness (as may occur in cancer) reduces the vibration amplitude. Thus, there is potential for CE-MMUS to achieve improved stiffness sensitivity. Our aim is to define the potential contribution of CEMMUS in colorectal cancer diagnosis and surgical guidance.
\end{abstract}

Keywords-magnetic microbubbles, contrast-enhanced magneto-motive ultrasound, elastography, colorectal cancer, SPION

Cancer Research UK

\section{INTRODUCTION}

Colorectal cancer is the 2 nd most common cause of cancer death in the UK, accounting for $10 \%$ of all cancer deaths (2016) [1] (2015) [2]. Treatment for rectal cancer often involves major resectional surgery that, in addition to carrying a significant risk of complications, may result in stoma; Both with the potential to substantially reduce a patient's quality of life. All patients undergo contrast CT and MRI imaging during pre-treatment staging, which remains limited in the detection and characterisation of lymph tissue. Furthermore, identification of the sentinel draining lymph, a key indicator of lymphatic spread is not possible leaving the surgeon to perform major surgery to ensure complete oncological treatment. Development of a sentinel node detection technique could potentially lead to targeted surgical resection, and improve quality of life, yet ensure effective oncological treatment.

Magneto-motive ultrasound (MMUS) offers a multimodal imaging alternative for sentinel lymph node detection [3]. Superparamagnetic iron oxide nanoparticles (SPIONs) drain through the lymph after intra-dermal injection and can be imaged using MRI. Excitation with a low frequency $(4-16 \mathrm{~Hz})$ electromagnet causes the SPIONS to oscillate, creating a low frequency tissue vibration that can be imaged using ultrasound (centre frequency 20-30 MHz). Despite the small amplitude of displacement involved (1-2 $\mu \mathrm{m}$ [4]), filtering for the known frequency of vibration provides a means of overcoming the diffraction limit of conventional ultrasound imaging such that tissues that have taken up SPIONs can be distinguished from their surroundings. Since the amplitude of tissue displacement will be influenced by stiffness, MMUS also has the potential to present diagnostic information. MMUS therefore has the 
potential to replace dye and radioactive tracers for lymph node identification during surgery. This work proposes the use of microbubbles (MB) labelled with SPIONS (SPION-MBs) in conjunction with MMUS to generate larger tissue displacements capable of improving sensitivity of the technique which we refer to as contrast enhanced magneto-motive ultrasound (CE-MMUS). SPION-MBs have previously been developed and demonstrated for use in targeted drug delivery [5] and targeted imaging, as they can be guided to a desired location with the use of a permanent magnet [6], and this background magnetic field has been found to enhance MB cavitation amplitudes [7, 8]. MB have already been demonstrated as an effective contrast agent for the lymph system via intra-dermal injection [9]. However, their behaviour under an oscillating magnetic field is yet to be studied. We investigate how SPION-MBs can be used to generate larger tissue displacements as compared to SPIONs alone.

\section{MethodS}

\section{A. Tissue Mimicking Material}

Tissue mimicking material (TMM) was prepared by mixing polyvinyl alcohol, PVA (molecular weight 89,000-98,000, 99\% hydrolyzed, Sigma-Aldrich UK company Ltd. Dorset, England), and $0.01 \%$ benzalkonium (Sigma-Aldrich UK company Ltd. Dorset, England) in deionized water kept below the boiling point until completely dissolved, and then left to cool. Two concentrations were prepared, five and ten weight percent of PVA. Samples were prepared by transferring one millilitre of PVA solution into cylindrical moulds, with internal diameter of $17 \mathrm{~mm}$. Three replicas were made of each concentration PVA, and $0.5 \mathrm{mg} / \mathrm{mL}$ of $10 \mathrm{~nm}$ diameter SPIONs (WHKS1S12, Liquid Research Ltd, Bangor, UK) was added and mixed to form a homogeneous distribution. The samples were then subjected to three $24 \mathrm{~h}$ freeze thaw cycles to form a cryogel (PVA-c) by crosslinking the polymer. The finished samples were stored in sealed plastic containers.

\section{B. Compression testing}

In order to evaluate the final stiffness, each sample was subjected to compression testing (Bose 3100, Framingham, Massachusetts, U.S.). The height was individually measured using a digital caliper, and the diameter assumed equal to the internal diameter of the mould. The samples were placed in contact with the load cell, and compressed by $0.01 \mathrm{~mm}$. After ten minutes, a linear ramp was applied, compressing the sample to $5 \%$ strain at a rate of $0.02 \mathrm{~mm}$ per second. Force as a function of displacement was exported and scaled by surface area and initial height to obtain stress and strain, from which Young's Modulus could be estimated using the slope of a linear regression.

\section{Magnetic Excitation}

The TMM samples containing SPIONs were subject to magnetic excitation from an in-house built electromagnetic solenoid, see Fig. 1. The predominating, axial force on a single SPION can be approximated by

$$
F_{n}=\frac{V_{m} \chi_{n}}{\mu_{0}} B_{z} \frac{\partial B_{z}}{\partial z}
$$

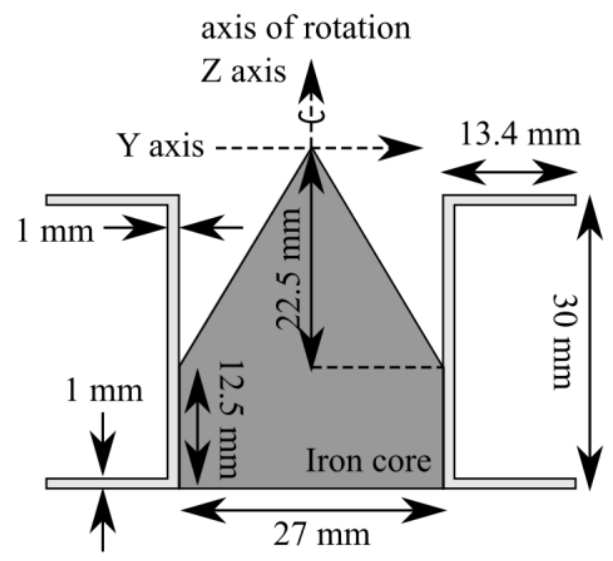

Figure 1. Cross-sectional schematic of the solenoid core (annealed soft iron, SEKELS GmbH) and spool. Spool fits 480 turns of $0.75 \mathrm{~mm}$ diameter enameled copper wire.

where $V_{m}$ is the magnetic volume of the SPION, $\chi_{n}$ is the magnetic susceptibility of the SPION, $\mu_{0}$ is the permeability of free space, and $B_{Z}$ is the magnitude of the magnetic flux density in the $\mathrm{Z}$ axis direction.

A $20 \mathrm{~Hz}$ sinusoidal signal was amplified (Behringer EP4000) and used to drive the solenoid. The vertical component of the output magnetic field was mapped using a Hall probe with active area $0.2 \times 0.2 \mathrm{~mm}$ connected to a gaussmeter (GM08, Hirst Magnetic Instruments Ltd, Cornwall, UK). A 2D scan over a 4x 4 $\mathrm{mm}$ area, covering the core tip was conducted in $0.25 \mathrm{~mm}$ increments, as well as a $6 \mathrm{~mm}$ vertical scan above the centre point.

\section{Laser Doppler Vibrometry}

Driving the solenoid at $20 \mathrm{~Hz}$ will generate a $40 \mathrm{~Hz}$ magnetic force acting on the magnetic nanoparticles, see equation 1 , but it can also create a mechanical vibration. In order to mechanically decouple the sample, it was placed on a holder just above the surface of the solenoid, underneath the laser Doppler vibrometer, LDV (Polytec, Waldbronn, Germany), scanning head. The laser spot was aligned with the core tip and the center of the sample before starting the measurement. A $3 \mathrm{~V}$ peak to peak signal, by $30 \mathrm{~dB}$, was used to drive the solenoid, a typical measurement ran for $7 \mathrm{~s}$. LDV measures velocity which was converted to displacement by integration.

\section{E. Finite Element Modelling}

A finite element modelling (FEM) software (COMSOL Multiphysics, Stockholm, Sweden) was used to simulate the effect of SPIONs organized in a bubble shell formation, as compared to homogeneously distributed. First, a model of the solenoid was constructed to simulate the field. Second, the computed field was used as an input to a second model containing either a single MB (diameter $1.2 \mu \mathrm{m}$, shell thickness $2 \mathrm{~nm}$ ) with magnetic properties confined to the shell, or a sphere of the same radius with the same amount of magnetic material distributed throughout the volume. Poisson's ratio was set to 0.499 , Young's modulus was $100 \mathrm{MPa}$ for air and $60 \mathrm{kPa}$ elsewhere, density of the surrounding material was $997 \mathrm{~kg} \mathrm{~m}^{-3}$, 
$1100 \mathrm{~kg} \mathrm{~m}^{-3}$ for the shell/sphere, and $11.2 \mathrm{~kg} \mathrm{~m}^{-3}$ for air. The electromagnetic properties used in the simulations are summarized in table 1 .

TABLE I. ELECTROMAGNETC PARAMETERS

\begin{tabular}{|l|c|c|c|}
\hline \multirow{2}{*}{ Material } & \multicolumn{3}{|c|}{ Material Property } \\
\cline { 2 - 4 } & $\begin{array}{c}\text { Permeability } \\
\text { (relative) }\end{array}$ & $\begin{array}{c}\text { Permittivity } \\
\text { (relative) }\end{array}$ & $\begin{array}{c}\text { Conductivity } \\
(\mathbf{S} / \mathbf{m} \text { ) }\end{array}$ \\
\hline Air & 1 & 1 & 0 \\
\hline Copper & 1 & 1 & $5.998 \times 10^{7}$ \\
\hline Iron & $2 \times 10^{6}$ & 1 & $1.12 \times 10^{7}$ \\
\hline
\end{tabular}

\section{RESULTS}

\section{A. Magnetic Field Mapping}

Measurements confirmed that the field was rotationally symmetrical, Fig. 2, with the peak value above the core tip of $1.38 \mathrm{~T}$ at a frequency of $20 \mathrm{~Hz}$ and $30 \mathrm{~dB}$ amplification. Furthermore, the field dropped off with vertical distance to the core tip, according to Fig. 4.

\section{B. Compression Testing}

The material response was predominantly linear at the rate and level of strain. Linear regression estimates of Young's Modulus, presented in Fig. 3, were $9.6 \pm 0.8$ for $5 \%$ PVA-c and $24.3 \pm 2.8$ for $10 \%$ PVA-c.

\section{Laser Doppler Vibrometry}

The largest vibration velocities and amplitudes were found at $40 \mathrm{~Hz}$, double the excitation frequency. Out of the 6 samples containing SPIONs, the signal to noise ratio in 1 of each PVA concentration was inadequate. The softer $5 \%$ PVAc samples display notably larger displacements, at $40 \mathrm{~Hz}$ the displacement of the $5 \%$ PVA-c samples were $426.9 \pm 9 \mu \mathrm{m}$ while the $10 \%$ PVA-c samples displaced on average $278 \pm 113 \mu \mathrm{m}$ at the same frequency.

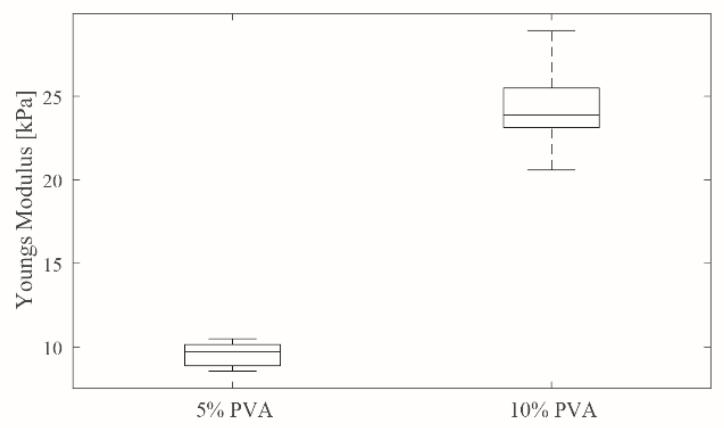

Figure 3. Box plot of estimated Young's modulus from the slope of regression lines. Compression testing was carried out directly after the samples had completely defrosted, and repeated 6 hours later.

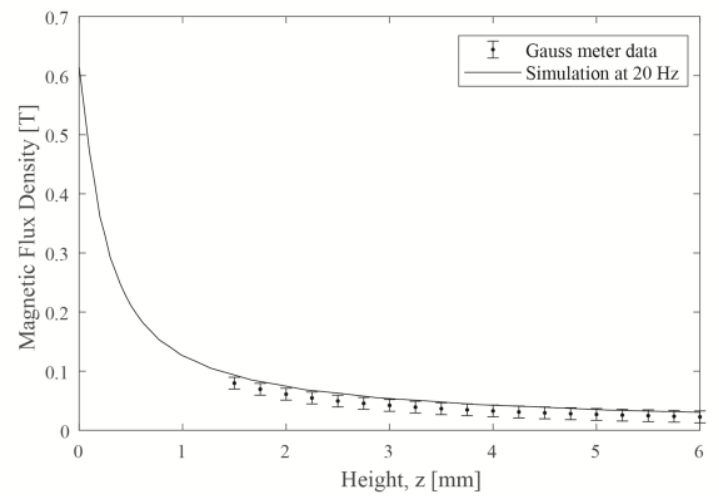

Figure 4. Magnetic flux density measured along the centre line of the solenoid and simulated. A $10 \mathrm{~dB}$ amplification was used.

\section{D. $\quad$ Finite Element Results}

The simulated field at $20 \mathrm{~Hz}$, Fig. 4, was similar to the measurement and 0.01 Thigher throughout along the centerline.

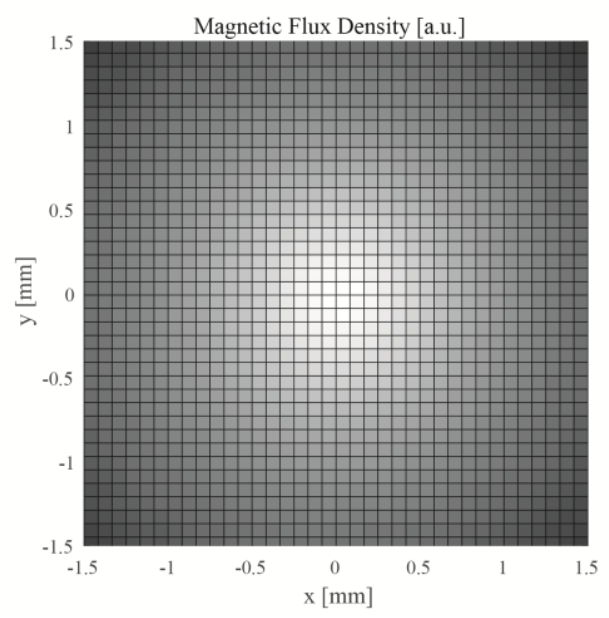

Figure 2. Normalized magnetic flux density measured above the core tip.

The second part of the simulation, comparing the displacement of a single solid sphere with that of a MB, indicate that the geometric configuration of the magnetic material has an effect on the resulting displacement. The simulated displacement was $2.3 x$ larger when the magnetic material was located in a shell as compared to homogeneously distributed.

\section{DISCUSSION AND CONCLUSION}

The results indicate that the development of $\mathrm{MBs}$ for MMUS could increase the vibration amplitude, and thereby also the sensitivity and range of tissue stiffness sensing.

The amplitude of vibration depends on the viscoelastic properties of the tissue, or in this case TMM. The displacement amplitude was found to be significantly smaller in the stiffer samples. The increase in Young's modulus from 9.6 to $24.3 \mathrm{kPa}$ (153\%), caused the displacement amplitude, as measured by LDV, to decrease by $35 \%$.

Our simulation results show that the organization of magnetic material in a MB shell affects the induced vibration. 
Furthermore, it suggests that the amplitude would be $2.3 \mathrm{x}$ larger in the MB case, as compared to a homogeneous distribution of magnetic material, which would directly affect the sensitivity. This implies that CE-MMUS would be more sensitive and would be capable of inducing a detectable movement in stiffer tissues, than MMUS using free SPIONs.

The different concentration of PVA in the TMMs were used to illustrate the relation between stiffness and vibration amplitude, but with only two concentrations, the results cannot be generalized. Furthermore, the analysis of the stress-strain data was done assuming a linear relation, and only extracting a single parameter, Young's modulus. While the linear assumption was valid in this regime, it is likely that mechanical non-linearity would influence the response at other frequencies. Therefore, characterizing the viscoelasticity would be preferable.

The excitation frequency of the solenoid was largely based on the sensitivity of the LDV system. In order to optimize the choice for future ultrasound applications, it is important to take the frequency response of the amplifier and solenoid into account, as well as heating of the solenoid. While the simulated field agreed well with the measurements at the settings used in this study, there are some discrepancies between the frequency response and linearity of the physical and the simulated system that would need to be accounted for, in order for the model to be valid at other driving currents.

Despite the driving frequency being selected with the LDV sensitivity in mind, the signal to noise ratio was only sufficient in four out of the six measurements. This could be due to the surface structure of the samples, and the ability to scatter light, causing the intensity of the returning laser reflection to be low. Using reflective paint on the top surface could alleviate this issue, and more repeats are necessary, in order to get more reliable measurements.

There are assumptions and simplifications in the model comparing MB to a solid sphere response. The mechanical parameters of the components are difficult to characterize, and depend on strain level, rate, temperature, ambient pressure, etc. The choice of these parameters influences the outcome in terms of vibration amplitude. Moreover, the susceptibility in equation $1, \chi_{n}$, is constant in the simulation, which is a valid assumption only when the magnetization of the particles is far from saturation.
The results are promising for the potential of CE-MMUS, indicating that smaller, stiffer inclusions can be made to oscillate, at an amplitude that is detectable by ultrasound.

\section{ACKNOWLEDGMENT}

The authors would like to acknowledge the help and support of the Medical and Industrial Ultrasonics group at the University of Glasgow. Special thanks to Andrew Reid and Grant Smillie at the University of Strathclyde for technical assistance.

\section{REFERENCES}

[1] Cancer Research UK, "Bowel cancer mortality statistics." [Online]. Available: https://www.cancerresearchuk.org/health-professional/cancerstatistics/statistics-by-cancer-type/bowel-cancer/mortality. [Accessed: 07-May-2019].

[2] Cancer Research UK, "Cancer incidence for common cancers." [Online]. Available: https://www.cancerresearchuk.org/health-professional/cancerstatistics/incidence/common-cancers-compared\#heading-Zero. [Accessed: 07-May-2019].

[3] M. Evertsson et al., "Multimodal Detection of Iron Oxide Nanoparticles in Rat Lymph Nodes Using Magnetomotive Ultrasound Imaging and Magnetic Resonance Imaging" IEEE Trans. Ultrason. Ferroelectr. Freq. Control, vol. 61, no. 8, pp. 1276-1283, 2014.

[4] Maria Evertsson, Pontus Kjellman, Magnus Cinthio, Roger Andersson, Thuy A Tran, Rene in't Zandt, Gustav Grafström, Hanna Toftevall, Sarah Fredriksson, Christian Ingvar, Sven-Erik Strand, Tomas Jansson, "Combined Magnetomotive ultrasound, PET/CT, and MR imaging of ${ }^{68} \mathrm{Ga}$-labelled superparamagnetic iron oxide nanoparticles in rat sentinel lymph nodes in vivo" Sci. Rep., vol. 7, no. 1, pp. 1-9, 2017.

[5] Helen Mulvana, Robert J Eckersley, Meng-Xing Tang, Quentin Pankhurst, Eleanor Stride, "Theoretical and Experimental Characterisation of Magnetic Microbubbles", Ultrasound in Medicine and Biology, vol.38, no.5, pp. 864-875, 2012.

[6] Helen Mulvana, Eleanor Stride, Richard J Browning, Tom Barack, MengXing Tang, Quentin Pankhurst, Jo V Hajnal, Dominic Wells, Robert J Eckersley, "Enhanced gene transfection in vivo using magnetic localisation of ultrasound contrast agents: preliminary results", IEEE International Ultrasonics Symposium, San Diego, 2010.

[7] Calum Crake, Joshua Owen, Sean Smart, Christian Coviello, ConstantinC. Coussios, Robert Carlisle, Eleanor Stride, "Enhancement and Passive Acoustic Mapping of Cavitation from Fluorescently Tagged Magnetic Resonance-Visible Magnetic Microbubbles In Vivo" Ultrasound Med. Biol., vol. 42, no. 12, pp. 3022-3036, 2016.

[8] J. Owen, Q. Pankhurst, and E. Stride, "Magnetic targeting and ultrasound mediated drug delivery: Benefits, limitations and combination", Int. J. Hyperth., vol. 28, no. 4, pp. 362-373, 2012.

[9] K. Cox, A. Severa, S. Jones, J. Weeks, P. Mills, H. Devalia, D. Fish, P. Jones, "Validation of a technique using microbubbles and contrast enhanced ultrasound (CEUS) to biopsy sentinel lymph nodes (SLN) in pre-operative breast cancer patients with a normal grey-scale axillary ultrasound”, Eur. J. Surg. Oncol., vol. 39, no. 7, pp. 760-765, 2013. 